\title{
Effect of ventilation therapy on mortality rate among obesity hypoventilation syndrome and obstructive sleep apnoea patients
}

\author{
Hanna-Riikka Kreivi (D, Tuomas Itäluoma (1) and Adel Bachour (1)
}

Affiliation: Sleep Unit, Dept of Respiratory Medicine, Heart and Lung Center, Helsinki University Hospital, University of Helsinki, Helsinki, Finland.

Correspondence: Hanna-Riikka Kreivi, Dept of Respiratory Medicine, Heart and Lung Center, Helsinki University Hospital, Haartmaninkatu 4, 00200 Helsinki, Finland. E-mail: hanna-riikka.kreiviahus.fi

\section{ABSTRACT}

Introduction: The prevalence of obesity is continually increasing worldwide, which increases the incidence of obesity hypoventilation syndrome (OHS) and its consequent mortality.

Methods: We reviewed the therapy mode, comorbidity and mortality of all OHS patients treated at our hospital between 2005 and 2016. The control group consisted of randomly selected patients with obstructive sleep apnoea (OSA) treated during the same period.

Results: We studied 206 OHS patients and 236 OSA patients. The OHS patients were older $(56.3$ versus 52.3 years, $\mathrm{p}<0.001$ ) and heavier (body mass index 46.1 versus $32.2 \mathrm{~kg} \cdot \mathrm{m}^{-2}, \mathrm{p}<0.001$ ), and the percentage of women was higher $(41.2 \%$ versus $24.2 \%, \mathrm{p}<0.001)$, respectively. The OHS patients had more hypertension $(83 \%$ versus $61 \%, \mathrm{p}<0.001)$ and diabetes $(62 \%$ versus $31 \%, \mathrm{p}<0.001)$ than the OSA patients, but no higher stroke ( $4 \%$ versus $8 \%, \mathrm{p}=0.058)$ or ischaemic heart disease $(14 \%$ versus $15 \%, \mathrm{p}=0.437)$ incidence. The 5- and 10-year, unadjusted survival rates were lower among the OHS patients than among the OSA patients ( $83 \%$ versus $96 \%$ and $74 \%$ versus $91 \%$, respectively; $\mathrm{p}<0.001$ ). Differences in mortality rates were not related to age, sex or body mass index; covariates such as Charlson Comorbidity Index and ventilation therapy predicted survival. The mortality rate decreased significantly $(p<0.001)$ both in OHS and OSA patients even after adjusting for covariates.

Conclusions: The mortality rate in OHS was significantly higher than that in OSA patients even after adjusting for covariates. Ventilation therapy by continuous positive airway pressure or noninvasive ventilation have reduced mortality significantly in all patients.

@ERSpublications

The mortality rate is significantly higher among OHS patients than OSA patients. Ventilation therapy significantly reduces mortality among both OHS and OSA patients. https://bit.ly/ 2ykGM31

Cite this article as: Kreivi H-R, Itäluoma T, Bachour A. Effect of ventilation therapy on mortality rate among obesity hypoventilation syndrome and obstructive sleep apnoea patients. ERJ Open Res 2020; 6: 00101-2019 [https://doi.org/10.1183/23120541.00101-2019].

Received: 22 April 2019 | Accepted after revision: 24 March 2020

Copyright $\odot$ ERS 2020. This article is open access and distributed under the terms of the Creative Commons Attribution Non-Commercial Licence 4.0. 


\section{Introduction}

Sleep-related hypoventilation includes a number of sleep-related hypoventilatory conditions, including obesity hypoventilation syndrome (OHS) [1]. OHS is defined by a resting daytime arterial carbon dioxide tension $\left(P_{\mathrm{aCO}_{2}}\right)$ of $\geqslant 6 \mathrm{kPa}$, a body mass index (BMI) of $\geqslant 30 \mathrm{~kg} \cdot \mathrm{m}^{-2}$ and the absence of an alternative cause of alveolar hypoventilation. Although not part of the diagnostic criteria, it is associated with nocturnal hypercapnia, nocturnal hypoxaemia and obstructive sleep apnoea (OSA) [2-5]. 90\% of OHS patients have concomitant OSA but some have only OHS with no night-time apnoea [6]. Although some of the underlying pathophysiology of hypoventilation for other diagnoses is better understood, the mechanisms of OHS remain incompletely characterised $[2,3]$. Multiple mechanisms have been implicated in the initiation and maintenance of chronic diurnal hypercapnia, including altered respiratory mechanics, compensatory metabolic derangements, OSA, hypothalamic dysfunction and hormonal influences $[7,8]$.

The exact prevalence of OHS is unclear but it is estimated to be approximately $0.15-0.6 \%$ of the general population [6,8-10]. OSA prevalence among women has been reported to be $2 \%$ and that among men, $4 \%$ [11]. Patients with OHS have higher morbidity and mortality than those with only OSA [12]. Although mortality is high among untreated patients with OHS, it falls with noninvasive ventilation (NIV) $[13,14]$.

The aim of this study was to evaluate the comorbidity, mortality and adherence to NIV therapy of our OHS patients, and to compare our results with those of patients referred to our clinic because of OSA.

\section{Material and methods \\ Study subjects}

We reviewed all the patients referred to our pulmonary clinic (a tertiary teaching university hospital) with an OHS diagnosis, defined as International Classification of Diseases (10th revision) diagnosis code E66.2 (morbid obesity with alveolar hypoventilation), between 2005 and 2016. The diagnostic criteria for OHS were a resting daytime $P_{\mathrm{aCO}}$ of $\geqslant 6 \mathrm{kPa}$ and a $\mathrm{BMI}$ of $\geqslant 30 \mathrm{~kg} \cdot \mathrm{m}^{-2}$ with no alternative cause for the hypercapnia. Obese patients with chronic respiratory failure or severe COPD with hypercapnia were not classified as OHS patients. We studied a total of 206 OHS patients.

As a control group, we randomly drew 236 patients from a sample of 3351 OSA patients followed at our clinic between 2005 and 2016.

\section{Sleep studies}

The baseline sleep studies were mainly conducted using a cardiorespiratory home polygraphy (Embletta; Embla, Denver, CO, USA). The definition of apnoea was the absence of nasal or buccal flow for $>10 \mathrm{~s}$ and the definition of hypopnea was a decrease of nasal flow of $\geqslant 50 \%$ for $\geqslant 10 \mathrm{~s}$ associated with a decrease in oxygen saturation of $\geqslant 3 \%$. Values of oxygen desaturation of $>4 \%$ per hour were also reported.

We drew an arterial blood sample while the patients were awake and in a supine position, having rested for $\geqslant 15 \mathrm{~min}$. The arterial blood sample was taken at the same hospital visit as the diagnostic sleep study. We immediately analysed the samples using a blood gas analyser (GEM Premier 4000 system; Instrumentation Laboratory, Bedford, MA, USA).

\section{Methods}

We collected data from hospital files on the patients' characteristics, comorbidity, medication, treatment, NIV and positive airway pressure (PAP) ventilation therapy, adherence to therapy, and mortality. We compared the two groups in terms of mortality, comorbidity and adherence to NIV or PAP therapy.

\section{NIV and PAP therapy}

For NIV therapy, Resmed VPAP devices (Resmed, San Diego, CA, USA) and Philips Respironics BiPAP devices (Philips Healthcare, Baltimore, MD, USA) were the primary treatment options for the OHS patients, whereas only Resmed devices were used for OSA patients.

PAP therapy was offered to patients with a respiratory event index (REI) of $>15 \mathrm{~h}^{-1}$ during the sleep study or patients with a REI of $5-14 \mathrm{~h}^{-1}$ and symptoms related to OSA. We started therapy with the automatic positive airway pressure (APAP) mode for the majority of our patients. The initiation of NIV therapy was supervised by an overnight oximetry and a transcutaneous capnograph (TCM-3 (Radiometer, Copenhagen, Denmark) or the Sentec Digital Monitoring System (Sentec AG, Therwil, Switzerland)). Every resident in Finland is covered by national insurance regardless of their employment situation. NIV and PAP therapy did not incur any additional costs for the patients.

We obtained data on NIV and PAP therapy and treatment adherence from the ResScan (Resmed) and Encore Pro (Philips Healthcare) programs. The first NIV follow-up visits were scheduled to the ward 
where the overnight oximetry-capnography was carried out. After the first follow-up visit, the next follow-up visit was typically scheduled at the outpatient clinic, and arterial blood gases and device download were carried out before the appointment. For PAP patients, we used device download as a follow-up method.

\section{Comorbidity}

We used the age-adjusted Charlson Comorbidity Index (CCI) to evaluate comorbidity [15], which consists of 19 medical conditions weighted from 1 to 6, with total scores ranging from 0 to 37 (0: no comorbidity). All data on patients' medical conditions were collected from the hospital files.

\section{Statistical analysis}

An independent samples t-test and Chi-squared test was used to compare the differences between groups. A Cox regression analysis was performed using several covariates to predict factors that influence mortality for OSA and OHS. A survival curve with and without adjustment to significant covariates was drawn. A p-value of $<0.05$ was considered significant. All statistical analyses were conducted using SPSS Statistics 25 for Windows (IBM, Armonk, NY, USA). We ignored any missing data and did not adjust for them. We also ignored missing follow-up data on NIV or PAP adherence. Data on mortality were obtained from the national registry, enabling us to gather survival data on all the included patients.

\section{Bias}

This study was performed in one centre, a university teaching hospital. We relied on the hospital record diagnoses.

\section{Study size}

We decided to include all accessible patients, starting from the date on which our hospital file was digitised in 2005 .

\section{Ethical considerations}

The study was carried out as a patient file study and no intervention was needed. This study was approved by the Heart and Lung Center of the Helsinki University Hospital (code \$14, 25 April 2017, permit number HUS/24/2017).

\section{Results}

Table 1 describes the characteristics of the patients. Compared to OSA patients, among the OHS patients, we found significantly more women; they were also older and heavier, and had higher comorbidity scores, more severe REI and higher $P_{\mathrm{aCO}_{2}}$. The mean \pm sD follow-up time was $9.1 \pm 4.8$ years (range 0.2-28.9 years).

\section{TABLE 1 Characteristics of obesity hypoventilation syndrome (OHS) and obstructive sleep} apnoea (OSA) patients

\begin{tabular}{|c|c|c|c|}
\hline & OHS & OSA & p-value \\
\hline Patients $\mathrm{n}$ & 206 & 236 & \\
\hline Women \% & $41.3 \%$ & $24.2 \%$ & $<0.001$ \\
\hline BMI $\mathrm{kg} \cdot \mathrm{m}^{-2}$ & 46.1 & 32.2 & $<0.001$ \\
\hline $\mathrm{CCl}$ score & 3.1 & 1.6 & $<0.001$ \\
\hline Age years & 56.3 & 52.3 & $<0.001$ \\
\hline$P_{\mathrm{aO}_{2}} \mathrm{kPa}$ & 7.9 & 10.2 & $<0.001$ \\
\hline$P_{\mathrm{acO}_{2}} \mathrm{kPa}$ & 7.0 & 5.3 & $<0.001$ \\
\hline Awake $S_{\mathrm{pO}_{2}}$ & $91.0 \%$ & $94.9 \%$ & $<0.001$ \\
\hline Mean $\mathrm{S}_{\mathrm{pO}_{2}}$ & $84.8 \%$ & $93.2 \%$ & $<0.001$ \\
\hline REI $h^{-1}$ & 60.1 & 32.3 & $<0.001$ \\
\hline $\mathrm{ODI}_{3} \mathrm{~h}^{-1}$ & 65.31 & 26.1 & 0.253 \\
\hline $\mathrm{ODI}_{4} \mathrm{~h}^{-1}$ & 61.3 & 29.8 & $<0.001$ \\
\hline ESS score & 10.7 & 9.2 & 0.033 \\
\hline
\end{tabular}

Data are presented as means unless otherwise stated. BMI: body mass index; $\mathrm{CCl}$ : Charlson Comorbidity Index; $P_{\mathrm{aO}_{2}}$ : arterial oxygen tension; $P_{\mathrm{acO}}$ : arterial carbon dioxide tension; $\mathrm{S}_{\mathrm{pO}_{2}}$ : oxygen saturation measured by pulse oximetry; REI: respiratory event index; $\mathrm{ODI}_{3}: \geqslant 3 \%$ oxygen desaturation index; $\mathrm{ODI}_{4}$ : $\geqslant 4 \%$ oxygen desaturation index; ESS: Epworth Sleepiness Scale. 
Out of 206 OHS patients, results of blood gas samples or overnight oximetry-capnography recording were available for 171 patients, and out of 236 OSA patients, for only 10 patients.

\section{Comorbidity}

The prevalence of asthma, COPD, high blood pressure, diabetes, atrial fibrillation and psychiatric diagnoses was significantly higher in the OHS group than the OSA group (table 2). However, smoking prevalence, coronary heart disease, hypercholesterolaemia and stroke history of the OHS and OSA patients did not differ statistically (table 2 ).

\section{Confounding factors for survival}

A Cox regression analysis showed that the CCI score, the presence of diabetes and the status of ventilation therapy (still on ventilation or abandoned) were significant predictors of survival in both OHS and OSA. Ventilation was by far the most predictive factor for ameliorating survival with an odds ratio of 13.2 times (table 3).

\section{Survival}

An unadjusted survival curve for the patients with OSA and the patients with OHS showed a significant reduction in survival among the OHS group compared to the OSA group $(p<0.001)$ (figure 1a). This difference was maintained after adjustment for covariates (figure 1b).

The 5- and 10-year unadjusted survival rates were $83 \%$ and $74 \%$, respectively, among those with OHS; and $96 \%$ and $91 \%$, respectively, among those with OSA.

We observed no significant sex-based difference in survival in either the OSA or OHS group. $\left(\chi^{2}(1\right.$, $\mathrm{n}=442)=0.541, \mathrm{p}=0.504)$.

\section{Effects of ventilation on survival}

In the OHS group, we found a significant $(\mathrm{p}<0.001)$ improvement in survival among patients who stayed on ventilation compared to those patients who abandoned ventilation therapy. This improvement was maintained after adjusting for covariates (figure 2). We noticed the same effect of ventilation on survival for OSA patients, with a less marked improvement in survival with ventilation that was, nevertheless, statistically significant $(\mathrm{p}<0.001)$ (figure 3$)$.

\section{NIV and PAP therapy adherence}

A total of 114 out of 206 patients remained on ventilation therapy at the final follow-up. The ventilation therapy adherence data on 34 patients were missing. The corresponding number for OSA was 115 out of 236 patients. Among the all 229 patients who remained on ventilation therapy, only eight patients had used their ventilation device $<70 \%$ of days, even though their survival rate was still significantly $(\mathrm{p}<0.001)$ better than those who totally abandoned ventilation therapy (figure 4). The mean \pm SD daily ventilation use did not differ significantly between the OHS and OSA patients: $7.04 \pm 3.16$ versus $7.50 \pm 2.17 \mathrm{~h} \cdot \mathrm{day}^{-1}$, respectively $(\mathrm{p}=0.238)$.

\section{TABLE 2 Comorbidities in obesity hypoventilation syndrome (OHS) and obstructive sleep}

\section{apnoea (OSA) groups}

\begin{tabular}{lccc} 
& OHS & OSA & p-value \\
\hline Nonsmokers & $53(31 \%)$ & $79(39 \%)$ & 0.203 \\
Smokers & $49(29 \%)$ & $45(22 \%)$ & 0.203 \\
Ex-smokers & $67(40 \%)$ & $80(39 \%)$ & 0.203 \\
COPD & $46(26 \%)$ & $12(6 \%)$ & $<0.001$ \\
Asthma & $50(28 \%)$ & $33(15 \%)$ & 0.001 \\
Diabetes & $110(62 \%)$ & $67(31 \%)$ & $<0.001$ \\
Hypertension & $148(83 \%)$ & $132(61 \%)$ & $<0.001$ \\
Hypercholesterolaemia & $82(46 \%)$ & $83(38 \%)$ & 0.066 \\
Atrial fibrillation & $38(21 \%)$ & $27(12 \%)$ & 0.012 \\
Psychiatric disorders & $47(26 \%)$ & $29(13 \%)$ & 0.001 \\
Stroke & $7(4 \%)$ & $18(8 \%)$ & 0.058 \\
Ischaemic heart disease & $25(14 \%)$ & $33(15 \%)$ & 0.437 \\
\hline Data are presented as n (\%), unless otherwise stated & & \\
\hline
\end{tabular}




\begin{tabular}{|c|c|c|c|c|c|c|}
\hline & $\boldsymbol{\beta}$ & SE & Wald test & df & p-value & $e^{\beta}(95 \% \mathrm{Cl})$ \\
\hline $\mathrm{CCl}$ score & 0.350 & 0.083 & 17.806 & 1 & $<0.001$ & $1.419(1.206-1.670)$ \\
\hline Asthma & 0.238 & 0.361 & 0.435 & 1 & 0.509 & $1.269(0.625-2.574)$ \\
\hline COPD & -0.187 & 0.406 & 0.212 & 1 & 0.645 & $0.829(0.374-1.837)$ \\
\hline Hypertension & 0.135 & 0.448 & 0.091 & 1 & 0.763 & $1.145(0.475-2.757)$ \\
\hline Ischaemic heart disease & 0.044 & 0.394 & 0.012 & 1 & 0.912 & $1.045(0.482-2.262)$ \\
\hline Diabetes & 0.820 & 0.408 & 4.044 & 1 & 0.044 & $2.269(1.021-5.044)$ \\
\hline Hypercholesterolaemia & -0.274 & 0.461 & 0.354 & 1 & 0.552 & $0.760(0.308-1.877)$ \\
\hline Metabolic syndrome & 0.015 & 0.494 & 0.001 & 1 & 0.975 & $1.015(0.386-2.674)$ \\
\hline Atrial fibrillation & 0.419 & 0.390 & 1.152 & 1 & 0.283 & $1.520(0.708-3.264)$ \\
\hline Psychiatric history & -0.322 & 0.377 & 0.730 & 1 & 0.393 & $0.724(0.346-1.517)$ \\
\hline Stroke & 0.447 & 0.844 & 0.281 & 1 & 0.596 & $1.564(0.299-8.186)$ \\
\hline Age & 0.016 & 0.018 & 0.819 & 1 & 0.365 & $1.016(0.981-1.053)$ \\
\hline Sex & -0.523 & 0.346 & 2.279 & 1 & 0.131 & $0.593(0.301-1.169)$ \\
\hline Body mass index & 0.044 & 0.023 & 3.731 & 1 & 0.053 & 1.045 (0.999-1.093) \\
\hline Ventilation therapy & 2584 & 0.443 & 33.947 & 1 & $<0.001$ & $13.246(5.554-31.589)$ \\
\hline
\end{tabular}

\section{Discussion}

The mortality rate was significantly higher among the OHS patients than the OSA patients even after adjustment for covariates. Ventilation therapy significantly reduced this high mortality among both OHS and OSA patients.

Our second main finding was that the OHS patients had higher comorbidity than the OSA patients. This high comorbidity was related to asthma, COPD, diabetes, hypertension, psychiatric disorders or atrial fibrillation. Surprisingly, neither the prevalence of smokers nor the incidence of stroke, hypercholesterolaemia or ischaemic heart diseases was significantly higher in the OHS group than in the OSA group

CASTRO-AnOn et al. [12] have previously shown that OHS patients have higher morbidity and mortality rates than OSA patients while undergoing treatment. They studied fewer OHS patients than we did in our study and reported a slightly higher 5-year OHS mortality rate of $15.1 \%$ than our finding of $8.4 \%$.

Information on the mortality rate of untreated or nonadherent OHS patients is scarce. NowBAR et al. [16] studied 47 OHS patients who were admitted to the medical services of three teaching hospitals and reported an 18 -month mortality of $23 \%$, substantially higher than our finding of $1.9 \%$. Effectively, the high
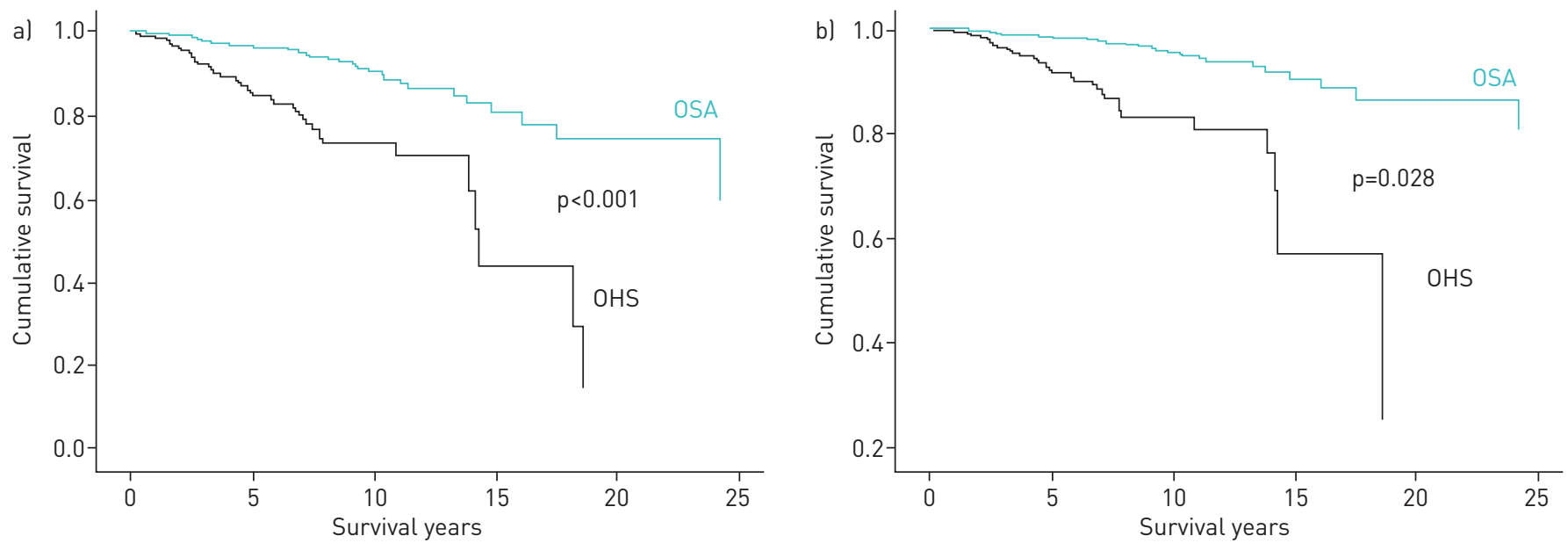

FIGURE 1 A survival curve of up to 25-year follow-up of obesity hypoventilation syndrome (OHS) and obstructive sleep apnoea (OSA) patients. a) No adjustment for covariates. b) Adjustment for Charlson Comorbidity Index, diabetes and ventilation therapy. 
FIGURE 2 A survival curve of patients with obesity hypoventilation syndrome according to their ventilation therapy status. Adjustment was performed for the Charlson Comorbidity Index.

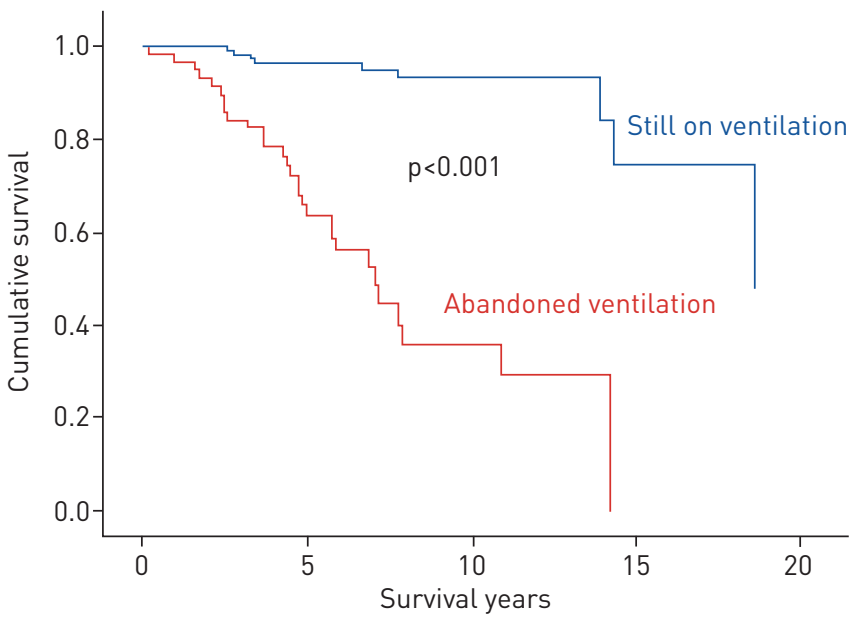

mortality rate that they reported could be explained by the critical medical condition of the patients in their study. Other authors have also reported a high 5-year mortality rate ranging from $15 \%$ to $30 \%$ among treated OHS patients $[11,14,17]$. In our study, the adjusted 5 -year mortality rate was $2 \%$ for patients with good therapy adherence and $27 \%$ for those with failed therapy. Our low mortality rate could be explained by the early diagnosis of OHS, full insurance coverage for all residents of Finland, and by providing NIV and PAP therapy equipment free of charge.

To our knowledge, no studies have previously compared the mortality of untreated OHS patients and untreated OSA patients. In our study, we found a significant difference between the mortality rate of OHS patients with failed/poor therapy adherence and those with OSA and failed/poor therapy adherence at 5and 10-year follow-up. Our results confirm the results of previous studies that showed mortality among untreated OHS patients is higher than that among treated OHS patients $[14,18]$.

One quarter of our OHS patients suffered from COPD and two thirds were active or ex-smokers. Several other authors excluded COPD patients from their studies related to OHS $[12,14,19]$. We chose not to exclude COPD patients from out study and as our data revealed over four-fold greater prevalence of COPD in the OHS group than the OSA group, despite equal prevalence of smoking and previous smoking, we admit there might have been overdiagnosis of COPD in our study population. The role of obesity in COPD is controversial. Its presence among patients hospitalised for exacerbations is associated with reduced in-hospital mortality and 30-day readmission rate [20, 21]. Meanwhile, other authors [22, 23] have reported increased respiratory-related mortality and healthcare utilisation among morbidly obese COPD patients. COPD patients mainly die because of pulmonary cancer or cardiovascular incidents, and had a mortality rate between $2 \%$ and $61 \%$ in a follow-up period from 1.1 to 14.5 years [24]. We do not believe that the relatively low mortality rate among our OHS patients is related to their high prevalence of COPD.

FIGURE 3 A survival curve of patients with obstructive sleep apnoea according to their ventilation therapy status. Adjustment was performed for the Charlson Comorbidity Index.

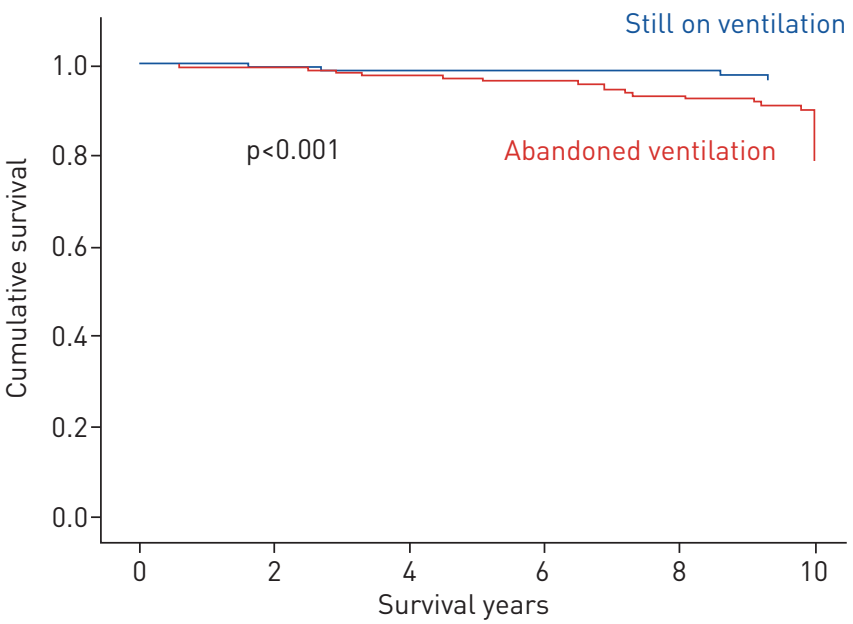


FIGURE 4 An adjusted survival curve for obesity hypoventilation syndrome and obstructive sleep apnoea patients according to ventilation status and percentage of days on which ventilation was used.

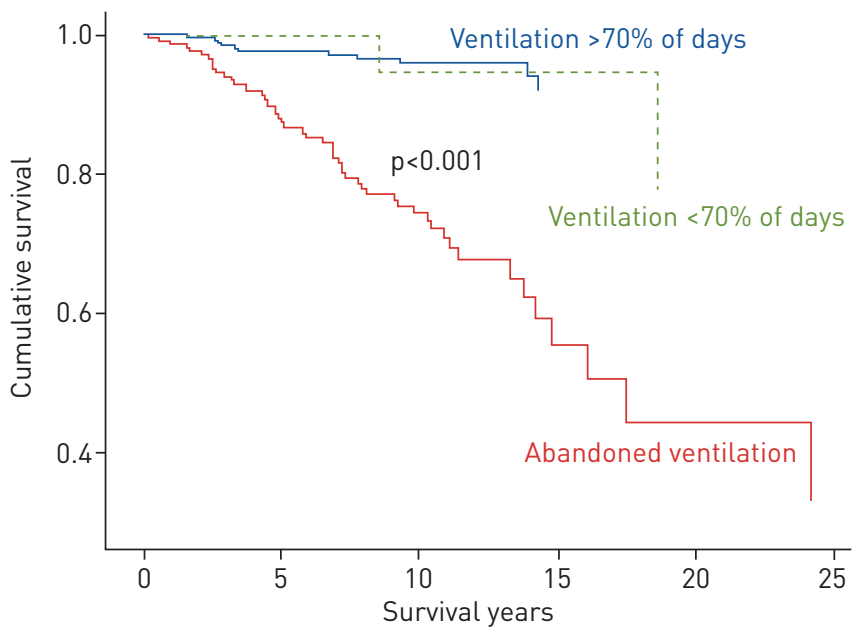

The prevalence of psychiatric illnesses was also significantly higher among OHS patients than among those with OSA $(26.4 \%$ versus $13.3 \%)\left(\chi^{2}(1, \mathrm{n}=396)=10.846, \mathrm{p}=0.001\right)$. We did not specify psychiatric diagnoses but it is known that severe obesity is common among patients with psychiatric diseases and medications. Psychiatric medications, such as the tranquilising effects of sleep medications and benzodiazepines, may have direct effects on breathing during sleep and may aggravate hypoventilation $[25,26]$.

Previously, it has been documented that if a patient suffers from both OSA and OHS, both NIV and PAP treatment can be beneficial and reduce carbon dioxide levels $[10,27,28]$. Follow-up studies are needed to investigate whether the two treatment modalities NIV (i.e. bilevel PAP) and continuous PAP or APAP differ in terms of mortality and cost-effectiveness. In our study, we primarily used NIV therapy for the OHS patients.

We noticed that the patients with OHS abandoned PAP therapy much less often than the OSA patients. For those who remained on PAP therapy, daily use did not differ between those with OHS and OSA. This difference in remaining on PAP therapy could be explained by more severe sleep respiratory disturbance indices among OHS patients than among OSA patients. In spite of the fact that the OSA patients abandoned PAP therapy more often than the OHS patients, their mortality rate remained significantly lower.

We reported a 5 -year mortality rate between $1 \%$ and $4 \%$ among the OSA patients, which is similar to previously reported rates $[29,30]$. The majority $(96.5 \%)$ of our patients who stayed on ventilation therapy used their device $>70 \%$ of days. Even with a frequency of use below this threshold, the benefit of ventilation on mortality maintained both in OHS and OSA patients. Our results agree with those of CAmpos-Rodriguez et al. [31], who reported no differences in 10-year mortality between OSA patients with daily PAP use of $>6 \mathrm{~h}$ and those with daily use of between 1 and $6 \mathrm{~h}$. Very recently, PATIL et al. [32] reported that a meta-analysis failed to demonstrate a clinically significant reduction in all-cause mortality with the use of PAP therapy among OSA patients.

Our study has some limitations. It was an epidemiological study, based on hospital file information. We performed no randomisation; therefore, our conclusion on the beneficial effect of NIV and PAP therapy on mortality should be taken with caution. Moreover, we did not report the cause of death, or the beneficial effect of PAP treatment regarding daytime sleepiness, blood gas values or quality of life, as other authors have done $[27,28,33]$. Our study was performed in one teaching hospital centre with a population that benefits from full medical coverage and medical ventilation equipment with no extra charges. Therefore, our findings may not apply to different insurance systems. We did not perform the propensity matching to compare the effect of covariates on mortality.

In conclusion, we showed that patients with OHS have a higher mortality rate than those with OSA and this finding was maintained after adjusting for covariates such as age, sex, BMI, comorbidity and ventilation therapy. We also showed that ventilation therapy significantly reduced all-cause mortality rates both in OHS and OSA patients. We recommend the regular use of ventilation therapy in these patients. 


\section{References}

1 American Academy of Sleep Medicine. International Classification of Sleep Disorders. 3rd Edn. Westchester, AASM, 2014.

2 Brown LK. Hypoventilation syndromes. Clin Chest Med 2010; 31: 249-270.

3 Piper AJ, Grunstein RR. Obesity hypoventilation syndrome: mechanisms and management. Am J Respir Crit Care Med 2011; 183: 292-298.

4 Javaheri S, Simbartl LA. Respiratory determinants of diurnal hypercapnia in obesity hypoventilation syndrome. What does weight have to do with it? Ann Am Thorac Soc 2014; 11: 945-950.

$5 \quad$ Piper AJ, Yee BJ. Hypoventilation syndromes. Compr Physiol 2014; 4: 1639-1676.

6 Balachandran JS, Masa JF, Mokhlesi B. Obesity hypoventilation syndrome epidemiology and diagnosis. Sleep Med Clin 2014; 9: 341-347.

7 Mokhlesi B, Tulaimat A. Recent advances in obesity hypoventilation syndrome. Chest 2007; 132: 1322-1336.

8 Kaw R, Hernandez AV, Walker E, et al. Determinants of hypercapnia in obese patients with obstructive sleep apnea: a systematic review and metaanalysis of cohort studies. Chest 2009; 136: 787-796.

9 Littleton SW, Mokhlesi B. The Pickwickian syndrome - obesity hypoventilation syndrome. Clin Chest Med 2009; 30: $467-478$

10 Masa JF, Pépin J-L, Borel J-C, et al. Obesity hypoventilation syndrome. Eur Respir Rev 2019; 28: 180097.

11 Young T, Palta M, Dempsey J, et al. The occurrence of sleep-disordered breathing among middle-aged adults. N Engl J Med 1993; 328: 1230-1235.

12 Castro-Anon O, de Llano LA P, Sanchez S DlF, et al. Obesity-hypoventilation syndrome: increased risk of death over sleep apnea syndrome. PLoS One 2015; 10: e0117808.

13 de Llano LA P, Golpe R, Ortiz Piquer M, et al. Short-term and long-term effects of nasal intermittent positive pressure ventilation in patients with obesity-hypoventilation syndrome. Chest 2005; 128: 587-594.

14 Priou P, Hamel JF, Person C, et al. Long-term outcome of noninvasive positive pressure ventilation for obesity hypoventilation syndrome. Chest 2010; 138: 84-90.

15 Sundararajan V, Henderson T, Perry C, et al. New ICD-10 version of the Charlson comorbidity index predicted in-hospital mortality. J Clin Epidemiol 2004; 57: 1288-1294.

16 Nowbar S, Burkart KM, Gonzales R, et al. Obesity-associated hypoventilation in hospitalized patients: prevalence, effects, and outcome. Am J Med 2004; 116: 1-7.

17 Budweiser S, Riedl SG, Jorres RA, et al. Mortality and prognostic factors in patients with obesity-hypoventilation syndrome undergoing noninvasive ventilation. J Intern Med 2007; 261: 375-383.

18 de Llano LA P, Golpe R, Piquer MO, et al. Clinical heterogeneity among patients with obesity hypoventilation syndrome: therapeutic implications. Respiration 2008; 75: 34-39.

19 Bouloukaki I, Mermigkis C, Michelakis S, et al. The association between adherence to positive airway pressure therapy and long-term outcomes in patients with obesity hypoventilation syndrome: a prospective observational study. J Clin Sleep Med 2018; 14: 1539-1550.

20 Lainscak M, von Haehling S, Doehner W, et al. Body mass index and prognosis in patients hospitalized with acute exacerbation of chronic obstructive pulmonary disease. J Cachexia Sarcopenia Muscle 2011; 2: 81-86.

21 Zapatero A, Barba R, Ruiz J, et al. Malnutrition and obesity: influence in mortality and readmissions in chronic obstructive pulmonary disease patients. J Hum Nutr Diet 2013; 26: Suppl. 1, 16-22.

22 Vozoris NT, O'Donnell DE. Prevalence, risk factors, activity limitation and health care utilization of an obese, population-based sample with chronic obstructive pulmonary disease. Can Respir J 2012; 19: e18-e24.

23 Jordan JG, Jr, Mann JR. Obesity and mortality in persons with obstructive lung disease using data from the NHANES III. South Med J 2010; 103: 323-330.

24 Sin DD, Anthonisen NR, Soriano JB, et al. Mortality in COPD: role of comorbidities. Eur Respir J 2006; 28 : $1245-1257$.

25 Gupta MA, Simpson FC. Obstructive sleep apnea and psychiatric disorders: a systematic review. J Clin Sleep Med 2015; 11: 165-175

26 Manthous CA, Mokhlesi B. Avoiding management errors in patients with obesity hypoventilation syndrome. Ann Am Thorac Soc 2016; 13: 109-114.

27 Howard ME, Piper AJ, Stevens B, et al. A randomised controlled trial of CPAP versus non-invasive ventilation for initial treatment of obesity hypoventilation syndrome. Thorax 2017; 72: 437-444.

28 Masa JF, Corral J, Alonso ML, et al. Efficacy of different treatment alternatives for obesity hypoventilation syndrome. Pickwick Study. Am J Respir Crit Care Med 2015; 192: 86-95.

29 Lee JE, Lee CH, Lee SJ, et al. Mortality of patients with obstructive sleep apnea in Korea. J Clin Sleep Med 2013; 9 : 997-1002.

30 Lavie P, Lavie L, Herer P. All-cause mortality in males with sleep apnoea syndrome: declining mortality rates with age. Eur Respir J 2005; 25: 514-520.

31 Campos-Rodriguez F, Pena-Grinan N, Reyes-Nunez N, et al. Mortality in obstructive sleep apnea-hypopnea patients treated with positive airway pressure. Chest 2005; 128: 624-633.

32 Patil SP, Ayappa IA, Caples SM, et al. Treatment of adult obstructive sleep apnea with positive airway pressure: an American Academy of Sleep Medicine systematic review, meta-analysis, and GRADE assessment. J Clin Sleep Med 2019; 15: 335-343.

33 Masa JF, Corral J, Caballero C, et al. Non-invasive ventilation in obesity hypoventilation syndrome without severe obstructive sleep apnoea. Thorax 2016; 71: 899-906. 